\title{
Rethinking How We Replace Knees and Hips
}

\section{Nouvelle approche pour le remplacement de la hanche et du genou}

\author{
by AlBERTA BONE AND JOINT HEALTH institute
}

$\mathrm{B}$ ud McLean and his wife, Geri, have travelled the World tOgether. When Bud's hip started slowing him down, he thought he knew what to expect in having it replaced, and was not looking forward to the process. A previous hip replacement in 1999 had left him in terrible shape. This time, though, he was pleasantly surprised. His care - including all aspects of pre-operative preparation, surgery, recovery and rehabilitation - was fully integrated and delivered by a multidisciplinary team. Bud was walking without support less than a month after being referred for surgery.

This Calgary resident's experience was thanks to the Alberta Hip and Knee Replacement Pilot Project - a radical new approach to providing joint replacements that dramatically reduced waiting times while improving patient care. The project proved so successful that the new model will be implemented all across Alberta. 


\section{A Growing Need}

Hip and knee replacements are a proven method of alleviating pain and restoring function and mobility. However, the care that patients receive can vary according to such factors as socio-economic status, age and geographic location. Wait times, both for a consultation and for

The KT Challenge

Improving patient care and reducing wait times for hip and knee replacements by implementing evidence-based health system changes surgery, are long. In Alberta, as in virtually every other jurisdiction in Canada, these wait times were currently beyond what is deemed optimal for most patients at the same time that the study was

initiated (WCWL Project 2003; ABJHI 2006). Alberta's aging population and growing proportion of seniors (Alberta Health and Wellness 2006), as well as rising rates of obesity (Statistics Canada 2005), indicate that the need for hip and knee replacements will grow.

In 2004, the Alberta Orthopaedic Society (AOS), Alberta Bone and Joint Health Institute $(\mathrm{ABJHI})$, the province's health regions and Alberta Health and Wellness decided to partner together to address the issues associated with hip and knee replacements. They agreed to redesign the continuum of care for these surgeries in Alberta and, in 2005, the partners launched a 12-month pilot project in three health regions to compare their new approach to conventional practice.

Five key groups were involved in the pilot project, each charged with specific responsibilities:

- Orthopaedic surgeons, whose practices and procedures would be altered and subject to standardization based on best available evidence;

- Family physicians, whose referral processes would be standardized and who would take on a more active role in their patients' pre-operative preparation and postoperative follow-up;

+ Health regions, which would establish evidence-based practices and procedures, providing the resources required to support them;

- Alberta Health and Wellness, which would provide funding for the necessary pilot resources and a policy environment to support the changes in the continuum of care for hip and knee replacements; and

- Patients, who would consent to participate in a randomized, controlled study throughout the pilot, involving intervention and control groups. 


\section{Setting a High Standard}

The partners based the pilot project on a framework of patient-centred principles:

- Deliver all services according to best benchmarks.

- Advance approaches to prevention, diagnosis and treatment based on informed decision-making, using a combination of the best available evidence and sound medical judgment.

- Continually evaluate all services for quality to ensure that value is always being created.

- Give referring physicians and patients access to the first available surgeon, or to the surgeon of their choice, recognizing that surgeon choice may mean a longer wait.

\section{Knowledge Translation Activities}

Knowledge users engaged in

- Shaping research questions

- Data collection and tool development

- Interpreting study findings

- Disseminating results

- Moving evidence into practice

- Widespread dissemination and application of knowledge
These principles could not have been applied, and the pilot could not have been successful, without constant interaction and communication among partners.

$\mathrm{ABJHI}$, together with the health regions, AOS, surgeons and physicians, developed the tools and processes required for a standardized hip and knee replacement care path that extended from primary care through to surgery, recovery and rehabilitation. The care path

included a consultation referral template, patient contracts, customized treatment plans, a patient optimization program, evidence-based clinical practices and procedures and scheduled patient follow-up.

Referral, diagnosis and treatment were all provided in multidisciplinary, community-based, single-purpose hip and knee replacement clinics. Health region clinical leaders, supported by ABJHI, trained hospital acute care and operating room staff in the new continuum, and engaged family physicians in the referral process.

$\mathrm{ABJHI}$, which served as the hub for the pilot, met regularly with surgeon groups, health regions and Alberta Health and Wellness department leaders to provide updates on progress, issues, interim results and proposed actions to remedy issues as they arose.

A provincial committee was formed to provide project oversight, while a working group representing all stakeholders managed activities and issues and kept the stakeholder groups informed of progress. One health region seconded an executive-level employee to act as project director. 


\section{How Did It Work?}

The pilot project, conducted in a protected research environment, improved patient care and outcomes and reduced wait times. Key results included the following:

- Hospital stays were reduced by almost a day and a half, from 6 days to 4.7 days.

- Eighty-five per cent of patients were up and mobile the day of their surgery.

- Patients were better able to function physically and had less pain following surgery.

- The wait to see a surgeon dropped from an average of 145 working days to 21 , and the wait from consultation to surgery fell from an average of 290 working days to 37.

- Patients and healthcare providers were more satisfied.

\section{Impact}

- Pilot results include reduction in wait to see a surgeon, reduction in length of hospital stay, increased patient/provider satisfaction, earlier mobility, improved physical function and less pain following surgery.

- The success of the pilot has led to province-wide implementation of the new continuum of care.
The partners caution that results such as these will be more difficult and take longer to achieve outside a controlled pilot environment where services are subject to normal dayto-day operational pressures. But the effort has begun.

Alberta Health and Wellness declared the pilot a "success story" and announced that the new continuum

would be implemented as the standard of care province-wide. Health and Wellness has committed funds to Alberta's health regions to support the transition from the former practices to the new continuum, and has provided ABJHI with funding to facilitate the province-wide implementation.

In the process of carrying out the pilot project, the partners learned a great deal about how to effect change in the healthcare system. Above all, they learned that problems such as wait times, service quality and system efficiency can be solved, however challenging the process.

The partners found that the key to the successful pilot was collaboration among all stakeholders. Change often meets with resistance, and physician affinity for independent practice makes team-based medicine difficult to implement. Attention must be paid to the different interests of all stakeholders, and everyone involved needs to define collaboratively the required resources, funding, testing period and standardized measurement framework.

Collaboration and consensus among partners are essential to gathering the evidence that is needed to underpin change, and are also critical to gaining acceptance by 
physicians and healthcare providers. Further, it's essential that healthcare administrators, policy makers and practitioners agree that practices, decisions and technologies used in healthcare delivery should be informed by the best available evidence, and that they must be willing to change based on new evidence. A system needs to be in place to gather data for monitoring and evaluation of system performance, to support continuous improvement and to provide the evidence that supports change.

Using these key tools - collaboration, evidence and a willingness to change - the new approach to hip and knee replacement could be applied to other areas of musculoskeletal care and provide a template for change that could be applied to other areas of healthcare all across Canada.

\section{REFERENCES}

Alberta Bone and Joint Health Institute (ABJHI). 2006. Canadian Institute of Health Research Knowledge Translation Casebook: "Rethinking How We Replace Knees and Hips." Retrieved May 31, 2009. <http://www.albertaboneandjoint.com>.

Alberta Health and Wellness. 2006 (July). Report on the Health of Albertans. Retrieved May 31, 2009. <http://www.health.alberta.ca/documents/Health-of-Albertans-2006.pdf>.

Statistics Canada Health Statistics Division. 2005 (July 6). Canadian Community Health Survey 2004: Obesity among Children and Adults. Retrieved March 31, 2009. <http://www.statcan.gc.ca/ daily-quotidien/050706/dq050706a-eng.htm>.

Western Canada Waiting List (WCWL) Project. 2003. Retrieved March 31, 2009. <http://www. wcwl.org >. 